\title{
NEW ANALYSIS OF WITTAKER FUNCTIONS
}

\author{
S.F. RAGAB
}

Summary. Integrals involving products of two Whittaker functions and Bessel functions are evaluated in $\S \S 3,4$. Also the integrals

$$
\int_{0}^{\infty} t^{\rho-1} W_{k_{1} m}(t) W_{-k_{1} m}(t) W_{\mu, \nu}\left(\frac{2 i z}{t}\right) W_{\mu, \nu}\left(\frac{-2 i z}{t}\right) d t
$$

and

$$
\int_{0}^{\infty} t^{\rho-1} W_{k_{1} m}(t)-W_{-k_{1} m}(t) W_{\mu, \nu}(2 z t) W_{-\mu, \nu}(2 z t) d t
$$

are evaluated in $\S 5$ while in $\S 6$ integrals involving the product of three Whittaker functions are established.

\section{$\S$ Introductory.}

Here integrals involging products of Whittaker functions will be established in $\S \S$ 4 and 5 . In $\S 3$ integrals involving Whittaker function will be established. They will be derived from subsidiary formulae which will be stated and proved in $\S 2$. These also will be deduced from the theory of MAC-ROBERTS' $E$-functions whose definitions and properties are given in [1] pp. 348-358). A brief account of the $E$-functions is given in ([2] pp 393). This is because that the Whittaker function is essentially ([1] p. 406) an $E$-function in virtue of the formula

$$
E\left(\frac{1}{2}-k+m, \frac{1}{2}-k-m: z\right)=\Gamma\left(\frac{1}{2}-k+m\right) \Gamma\left(\frac{1}{2}-k-m\right) z^{-k} e^{\frac{1}{2} z} W_{k_{1} m}(z)
$$

where $W_{k_{1} m}(z)$ is the known WHITTAKER function.

The following formulae are required in the proofs: ([3], p. 432):

$$
\begin{aligned}
& \int_{0}^{\infty} t^{p-1} W_{k_{1} m} W_{-k_{1} m}(t) E\left(p ; \alpha_{r}: q ; \rho_{s}: z / t^{2}\right) d t \\
= & 2^{\rho-1} \pi^{-\frac{1}{2}} E\left(\begin{array}{c}
\alpha_{1}, \ldots, \alpha_{p}, \frac{1}{2}+\frac{1}{2} \rho-m, \frac{1}{2}+\frac{1}{2} \rho+m, \frac{1}{2}+\rho, 1+\frac{1}{2} \rho: z / 4 \\
\rho_{1}, \ldots, \rho_{q}, 1+\frac{1}{2} \rho-k, 1+\frac{1}{2} \rho+k .
\end{array}\right)
\end{aligned}
$$


where

$$
R(\rho \pm 2 m)>-1, R(\rho)>-1,|\operatorname{amp} z|<\frac{1}{2}(p-q+1) \pi, \text { and } p \geq q+1
$$

([3], p. 430):

$$
\begin{gathered}
E(\alpha, \beta:: z) E(1-\alpha, 1-\beta:: z)=\Gamma(\alpha) \Gamma(\beta) \Gamma(1-\alpha) \Gamma(1-\beta) \pi^{-\frac{1}{2}} \\
\left(\frac{1}{2} z\right)^{1-\alpha-\beta} \times \frac{1}{2 \pi} \sum_{i,-i} \frac{1}{i} E\left(\alpha, \beta, \frac{1}{2} \alpha+\frac{1}{2} \beta, \frac{1}{2} \alpha+\frac{1}{2} \beta+\frac{1}{2}: \alpha+\beta: e^{i \pi} \frac{z^{2}}{4}\right)
\end{gathered}
$$

([1], p. 395):

$$
\begin{aligned}
W_{k_{1} m}(i z) W_{k_{1} m}(-i z) & =\frac{z^{2 k}}{2^{2 k} \Gamma\left(\frac{1}{2}\right) \Gamma\left(\frac{1}{2}-k+m\right) \Gamma\left(\frac{1}{2}-k-m\right)} \\
& \times E\left[\frac{1}{2}-k+m, \frac{1}{2}-k, 1-k: 1-2 k: \frac{1}{4} z^{2}\right],
\end{aligned}
$$

$([1]$, p. 406 ex. 28):

$$
\frac{z^{\alpha+\beta-1} e^{-z}}{\Gamma(1-\alpha) \Gamma(1-\beta)} E\left(1-\alpha, 1-\beta:{ }^{\prime} z\right)=\frac{1}{2 \pi} \sum_{i,-i} \frac{1}{i} E\left(\alpha, \beta:: e^{i \pi} z\right)
$$

where the symbol $\Sigma_{i,-i}$ means that in the expression following it $i$ is to be replaced by $-i$ and the two espression are to be added.

$([4], \mathrm{p}, 304)$ :

$$
z^{-\lambda} \sum_{i,-i} \frac{1}{i} E\left(P ; \alpha_{r}+\lambda, 1: q ; \rho_{s}+\lambda: e^{i \pi} z\right)=\sum_{i,-i} \frac{1}{i} E\left(P ; \alpha_{r}, 1: q ; \rho_{s}: e^{i \pi} z\right)
$$

([5], p. 759):

$$
\begin{aligned}
\sum_{i,-i} \frac{1}{i}\left(P ; \alpha_{r}: q ; \rho_{s}: z e^{i \pi}\right) & =(2 \pi)^{-\frac{1}{2}(n-1)(p-q-2)} n^{\Sigma \alpha_{r}-\Sigma \rho_{s}-\frac{1}{2}(p-q-1)} \\
& \times \sum_{i,-i} \frac{1}{i} E\left[\begin{array}{c}
\Delta\left(\alpha_{1}, n\right), \ldots, \Delta\left(\alpha_{p} ; n\right):\left(z / n^{p-q-1}\right)^{n} e^{i \pi} \\
\frac{1}{n}, \frac{2}{n}, \ldots, \frac{n-1}{n}, \Delta\left(\rho_{1} ; n\right), \ldots, \Delta\left(\rho_{q} ; n\right)
\end{array}\right]
\end{aligned}
$$

where $p>q+1, n$ is any positive integer, $|\operatorname{amp} z|<\frac{\pi}{2}(p-q-1)$ and the symbol $\Delta(n ; \alpha)$ represents the set of parameters

$$
\frac{\alpha}{n}, \frac{\alpha+1}{n}, \ldots, \frac{\alpha+n-1}{n}
$$


The integrals, involving WHITTAKER functions will be deduced from the following integrals involving $E$-function

([1], p. 406, ex. 30):

$$
\int_{0}^{\infty} e^{-\lambda} \lambda^{\rho-1} E\left(p ; \alpha_{r}: q, \rho_{s}: \frac{z}{\lambda^{n}}\right) d \lambda=(2 \pi)^{\frac{1}{2}-\frac{1}{2} n} n^{\rho-\frac{1}{2}} \times E\left(P+n ; \alpha_{r}: q ; \rho_{s}: z / n^{n}\right),
$$

where $n$ is any positiove integer, $R(\rho)>0$,

$$
=\alpha_{\rho+v+1}=(\rho+v) / n,(v=0,1,2, \ldots, n-1) .
$$

$([6], \mathrm{p} .77)$ :

$$
\begin{aligned}
& \int_{0}^{\infty} e^{-\lambda} \lambda^{\rho-1} E\left(P ; \alpha_{r}: q ; \rho_{s} z \lambda^{n}\right) d \lambda=\pi \operatorname{cosec}(\rho \pi)(2 \pi)^{\frac{1}{2} n-\frac{1}{2}} n^{\rho-\frac{1}{2}} \\
& E\left(P ; \alpha_{r}: 1-\frac{\rho}{n}, 1-\frac{\rho+1}{n}, \ldots, 1-\frac{\rho+n-1}{n}, q ; \rho_{s} ; e^{ \pm i n \pi} n^{n} z\right) \\
& +2^{\frac{1}{2}-\frac{1}{2} n} \pi^{\frac{1}{2}+\frac{1}{2} n} \sum_{v=0}^{n-1}(-1)^{v+1} \frac{n^{-\frac{1}{2}-v} z^{-(\rho+v) / n}}{\sin \left(\frac{\rho+v}{n}\right) \pi \Pi_{s=1}^{v} \sin \frac{s \pi}{n} \Pi_{t=1}^{n-v-1} \sin \frac{t \pi}{n}} \\
& \times E\left[\begin{array}{c}
\alpha_{1}+\frac{\rho+v}{n}, \ldots, \alpha_{p}+\frac{\rho+v}{n}: e^{ \pm n \pi i} n^{n} z \\
\left.1+\frac{\rho+v}{n}, 1+\frac{1}{n}, \ldots, 1+\frac{v}{n}, 1-\frac{1}{n}, \ldots, 1-\frac{n-v-1}{n}, q: \rho_{s}+\frac{\rho+v}{n}\right]
\end{array}\right.
\end{aligned}
$$

where $n$ is any positive integer, $|\operatorname{amp} z|<\pi$ and

$$
R\left(m \alpha_{r}+\rho\right)>0, r=1,2, \ldots, p .
$$

([7], p. 258):

$$
4 \int_{0}^{\infty} \lambda^{P-1} K_{n}(2 \lambda) E\left(P ; \alpha_{r}: q ; \rho_{s}: \frac{z}{\lambda^{z}}\right) d \lambda=E\left(P+2 ; \alpha_{r}: q ; \rho_{s} ; z\right) \text {, }
$$

where

$$
R(\rho \pm n)>0, \alpha_{p+1}=(\rho+n) / 2 \text { and } \alpha_{p+2}=(\rho-n) / 2
$$

([8], p. 8):

$$
\begin{aligned}
& 4 i \pi \int_{0}^{\infty} \lambda^{\rho-1} J_{n}(2 \lambda) E\left(P ; \alpha_{r}: q ; \rho_{s}: z / \lambda^{2}\right) d \lambda \\
= & i^{\rho-n} E\left(P+2 ; \alpha_{r}: q ; \rho_{s}: z e^{-i \pi}\right)-i^{n-\rho} E\left(P+2 ; \alpha_{r}: q ; \rho_{s} z e^{i \pi}\right),
\end{aligned}
$$

where

$R(\rho+n)>0, R\left(\frac{3}{2}-\rho+2 \alpha_{r}\right)>0, r=1,2, \ldots, P, \alpha_{p+1}=\frac{\rho+n}{2}$ and $\alpha_{p+z}=(\rho-u) / 2$. 
([9], p.52):

$$
\begin{aligned}
& \int_{0}^{\infty} \lambda^{\rho-1} K_{n}(\lambda) E\left(P: \alpha_{r}: q ; \rho_{s}: z \lambda^{2}\right) d \lambda \\
= & 2^{\rho-2} \frac{\pi^{2}}{\sin \left(\frac{\rho+n}{2}\right) \sin \left(\frac{\rho-n}{2} \pi\right)} E\left(\alpha_{1}, \ldots, \alpha \rho ; 1-\frac{\rho+n}{2}, 1-\frac{\rho-n}{2}, \rho_{1}, \ldots, \rho_{q}: 4 z\right) \\
+ & \sum_{n,-n} \frac{\pi^{2}}{\sin \left(\frac{\rho+n}{2} \pi\right) \sin \pi n} \cdot z^{-\frac{\rho+n}{2}} E\left(\begin{array}{c}
\alpha_{1}+\frac{\rho+n}{2}, \ldots, \alpha_{p}+\frac{\rho+n}{2}: 4 z \\
1+\frac{\rho+n}{2}, 1+n, \rho_{1}+\frac{\rho+n}{2}, \ldots, \rho_{s}+\frac{\rho+n}{2}
\end{array}\right)
\end{aligned}
$$

where

$$
p \geq q+1, R\left(\rho \pm n+2 \alpha_{r}\right)>0, r=1,2, \ldots, p,|\operatorname{amp} z|<\pi .
$$

([10], p. 82):

$$
\begin{aligned}
& \int_{0}^{\infty} e^{-u} I_{n}(u) u^{\rho-1} E\left(p ; \alpha_{r}: q ; \rho_{s}: z / \mu^{2}\right) d u \\
= & \frac{\sin (\rho-n) \pi}{(2 \sqrt{2}) \cos (\rho \pi)} E\left[\begin{array}{c}
\left.\alpha_{1}, \ldots, \alpha_{p}, \frac{\rho+n}{2}, \frac{\rho-n}{2}, \frac{\rho+n+1}{2} \frac{\rho-n+1}{2}: z\right] \\
\frac{3}{4}+\frac{1}{2} \rho, \frac{1}{4}+\frac{1}{2} \rho, \rho_{1}, \ldots, \rho_{q}
\end{array}\right] \\
- & \frac{\cos (n \pi)}{(4 \sqrt{2} \cdot \pi) \sin \left(\frac{\rho}{2}-\frac{1}{4}\right) \pi} \cdot z^{\frac{\rho}{2}-\frac{1}{4}} \\
E & {\left[\begin{array}{c}
\left.\alpha_{1}+\frac{1}{4}-\frac{\rho}{2}, \ldots, \alpha_{p}+\frac{1}{4}-\frac{\rho}{2}, \frac{1}{4}+\frac{n}{2}, \frac{3}{4}+\frac{n}{2}, \frac{1}{4}-\frac{n}{2}, \frac{3}{4}-\frac{n}{2}: z\right] \\
-\frac{\cos (n \pi)}{4}-\frac{\rho}{2}, \frac{1}{2}, \rho_{1}+\frac{1}{4}-\frac{\rho}{2}, \ldots, \rho_{q}+\frac{1}{4}-\frac{\rho}{2}
\end{array}\right] } \\
& {\left[\begin{array}{c}
(4 \sqrt{2} \cdot \pi) \sin \left(\frac{3}{4}-\frac{\rho}{2}\right) \pi \\
z^{\frac{\rho}{2}-\frac{3}{4}}
\end{array}\right] } \\
& {\left[\begin{array}{c}
\left.\alpha_{1}+\frac{3}{4}-\frac{\rho}{2}, \ldots, \alpha_{p}+\frac{3}{4}-\frac{\rho}{2}, \frac{3}{4}+\frac{n}{2}, \frac{5}{4}+\frac{n}{2}, \frac{5}{4}-\frac{n}{2}: z\right] \\
\frac{7}{4}-\frac{\rho}{2}, \frac{3}{2}, \rho_{1}+\frac{3}{4}-\frac{\rho}{2}, \ldots, \rho_{q}+\frac{3}{4}-\frac{\rho}{2}
\end{array}\right] }
\end{aligned}
$$

where

$$
R(\rho+n)>0, R\left(2 \alpha r-\rho+\frac{1}{2}\right)>0 r=1,2,3, \ldots, P,|\operatorname{amp} z|<\pi
$$


([10] p. :

$$
\begin{aligned}
& \int_{0}^{\infty} e^{-u} I_{n}(u) u^{\rho-1}\left(P ; \alpha_{r}: q ; \rho_{s}: z u^{2}\right) d u \\
& =\frac{\pi}{\sqrt{2} \sin (\rho+n) \pi} E\left[\begin{array}{c}
\alpha_{1}, \ldots, \alpha_{p}, \frac{1}{4}-\frac{\rho}{2}, \frac{3}{4}-\frac{\rho}{2}: z \\
1-\frac{\rho+n}{2}, \frac{1}{2}-\frac{\rho+n}{2}, \rho_{1}, \ldots, \rho_{q}, 1+\frac{n-\rho}{2}, \frac{1}{2}+\frac{n-\rho}{2}
\end{array}\right] \\
& -\frac{\pi z^{-\left(\frac{\rho+n}{2}\right)}}{2 \sqrt{2} \cdot \sin \left(\frac{\rho+n}{2}\right)} E\left[\begin{array}{c}
\alpha_{1}+\frac{\rho+n}{2}, \ldots, \alpha_{p}+\frac{\rho+n}{2}, \frac{1}{4}+\frac{n}{2}, \frac{3}{4}+\frac{n}{2}: z \\
1+\frac{\rho+n}{2}, \frac{1}{2}, \rho_{1}+\frac{\rho+n}{2}, \ldots, \rho_{q}+\frac{\rho+n}{2}, 1+n, \frac{1}{2}+n
\end{array}\right] \\
& -\frac{\pi z^{-\left(\frac{e+n+1}{2}\right)}}{2 \sqrt{2} \cdot \cos \left(\frac{\rho+n}{2} \pi\right)} \\
& E\left[\begin{array}{c}
\alpha_{1}+\frac{\rho+n+1}{2}, \ldots, \alpha_{p}+\frac{\rho+n+1}{2}, \frac{3}{4}+\frac{n}{2}, \frac{5}{4}+\frac{n}{2}: z \\
1+\frac{\rho+n+1}{2}, \frac{3}{2}, \rho_{1}+\frac{\rho+n+1}{2}, \ldots, \rho_{q}+\frac{\rho+n+1}{2}, \frac{3}{2}+n, 1+n
\end{array}\right]
\end{aligned}
$$

where

$$
R\left(n+\rho+2 \alpha_{r}\right)>0, r=1,2, \ldots, p, R\left(\frac{1}{2}-\rho\right)>0,|\operatorname{amp} z|<\pi
$$

\section{§2. Subsidiary theorems.}

The theorems to be proved are

$$
\begin{gathered}
z^{l} e^{-\frac{1}{2} z} W_{k_{1} m}(z)=\frac{1}{2 \pi} \sum_{i,-i} \frac{1}{i} E\left[\begin{array}{c}
\left.\frac{1}{2}+m+l, \frac{1}{2}-m+l, 1: z e^{i \pi}\right] \\
1-k+l
\end{array}\right] \\
z^{l} e^{-\frac{1}{2} z} W_{k_{1} m}(z)=(2 \pi)^{-\frac{1}{2}(n-1)} n^{k+l+\frac{1}{2}} \\
\frac{1}{2 \pi} \sum_{i,-i} \frac{1}{i} E\left[\Delta\left(n ; \frac{1}{2}+m+l\right), \Delta\left(n ; \frac{1}{2}-m+l\right):\left(\frac{z}{n}\right)^{n} e^{i \pi}\right] \\
\Delta(n ; 1-k+l)
\end{gathered}
$$

where $n$ is any positive integer an the symbol $\Delta$ has the same meaning as in (7).

$$
\left(\frac{z}{2}\right)^{l} W_{k_{1} m}(z) W_{-k_{1} m}(z)=\pi^{-\frac{1}{2}}(2 \pi)^{-n} n^{\frac{1}{2}+2 l-2 k}
$$




$$
\times \sum_{i,-i} \frac{1}{i} E\left[\begin{array}{c}
\Delta\left(n ; \frac{1}{2}+\frac{1}{2} l\right), \Delta\left(n ; 1+\frac{1}{2} l\right), \Delta\left(n ; \frac{1}{2} l+m+\frac{1}{2}\right) \\
\Delta\left(n ; \frac{1}{2} l-m+\frac{1}{2}\right):\left(\frac{z}{4 n^{2}}\right)^{n} e^{i \pi} \\
\Delta\left(n ; 1+k+\frac{1}{2} l\right), \Delta\left(n ; 1+k-\frac{1}{2} l\right)
\end{array}\right]
$$

where $n$ is any positive integer, and the symbols $\Delta$ and $\Sigma_{i,-i}$ have the previons meanings.

Proof of (16). In (5), take

$$
\alpha=\frac{1}{2}+k+m, \beta=\frac{1}{2}+k-m,
$$

apply (1) and get

$$
\begin{aligned}
z^{k} e^{-\frac{1}{2} z} W_{k_{1} m}(z) & =\frac{1}{2 \pi} \sum_{i,-i} \frac{1}{i} E\left(\frac{1}{2}+k+m, \frac{1}{2}+k-m:: z e^{i \pi}\right) \\
& =\frac{1}{2 \pi} \sum_{i,-i} \frac{1}{i} E\left(\frac{1}{2}+k+m, \frac{1}{2}+k-m, 1: 1: z e^{i \pi}\right) .
\end{aligned}
$$

Here multiply both rides by $z^{l-k}$ and get

$$
\begin{aligned}
z^{l} e^{-\frac{1}{2} z} W_{k_{1} m}(z) & =\frac{1}{2 \pi} z^{l-k} \sum_{i,-i} \frac{1}{i} E\left(\frac{1}{2}+k+m, \frac{1}{2}+k-m, 1: 1: z e^{i \pi}\right) \\
& =\frac{1}{2 \pi} \sum_{i,-i} \frac{1}{i} E\left(\frac{1}{2}+m+l, \frac{1}{2}-m+l, 1: 1-k+l: z e^{i \pi}\right) \text { by }(6)
\end{aligned}
$$

This is formula (15). Formula (16) follows immediately from (15) by applying (7).

Proof of (17). To prove (17), take in (3)

$$
\alpha=\frac{1}{2}+k+m, \beta=\frac{1}{2}+k-m
$$

apply (1) and get

$$
\begin{aligned}
\left(\frac{z}{2}\right)^{2 k} W_{k_{1} m}(z) W_{-k_{1} m}(z) & =\pi^{-\frac{1}{2}} \frac{1}{2 \pi} \sum_{i,-i} \frac{1}{i} \\
& E\left[\begin{array}{c}
\left.k+\frac{1}{2}, k+1, \frac{1}{2}+m+k, \frac{1}{2}-m+k: \frac{e^{i \pi} z^{2}}{4}\right] \\
1+2 k
\end{array}\right]
\end{aligned}
$$


Here multiply by $\left(\frac{z^{2}}{4}\right)^{\frac{1}{2} l-k}$ and get

$$
\begin{aligned}
& \left(\frac{z}{2}\right)^{l} W_{k_{1} m}(z) W_{-k_{1} m}(z) \\
& =\pi^{-\frac{1}{2}} \frac{(z / 2)^{l-2 k}}{2 \pi} \sum_{i,-i} \frac{1}{i} E\left[\begin{array}{c}
\left.k+\frac{1}{2}, k+1, \frac{1}{2}+m+k, \frac{1}{2}-m+k: e^{i \pi} \frac{z^{2}}{4}\right] \\
1+2 k
\end{array}\right] \\
& =\pi^{-\frac{1}{2}}(z / 2)^{l-2 k} \times \frac{1}{2 \pi} \sum_{i,-i} \frac{1}{i} E\left[\begin{array}{c}
k+\frac{1}{2}, k+1, \frac{1}{2}+m+k, \frac{1}{2}-m+k, 1: e^{i \pi} \frac{z^{2}}{4} \\
1,1+2 k
\end{array}\right] \\
& =\pi^{-\frac{1}{2}} \sum_{i,-i} \frac{1}{i} E\left[\begin{array}{c}
\frac{1}{2}+\frac{1}{2} l, 1+\frac{1}{2} l, 1, \frac{1}{2} l+m+\frac{1}{2}, \frac{1}{2} l-m+\frac{1}{2}: e^{i \pi} \frac{z^{2}}{4} \\
1+k+\frac{1}{2} l, 1+k-\frac{1}{2} l
\end{array}\right]
\end{aligned}
$$

Thus we have

$$
\begin{aligned}
& \left(\frac{z}{2}\right)^{l} W_{k_{1} m}(z) W_{-k_{1} m}(z)=\pi^{-\frac{1}{2}} \frac{1}{2 \pi} \sum_{i,-i} \frac{1}{i} \\
& E\left[\begin{array}{c}
\frac{1}{2}+\frac{1}{2} l, 1+\frac{1}{2} l, 1, \frac{1}{2} l+m+\frac{1}{2}, \frac{1}{2} l-m+\frac{1}{2}: e^{i \pi} \frac{z^{2}}{4} \\
1+k+\frac{1}{2} l, 1+k-\frac{1}{2} l
\end{array}\right]
\end{aligned}
$$

Again formula (17) follows from (19) by applying (7).

\section{$\S 3$. Integrals involving one Whittaker functions:}

From 8 (with $n=1$ ) and (1) one gets

$$
\begin{aligned}
& \int_{0}^{\infty} \exp \left(-\lambda+\frac{z}{2 \lambda}\right) \lambda^{\rho+k-1} W_{k_{1} m}\left(\frac{z}{\lambda}\right) d \lambda \\
= & \frac{z^{k}}{\Gamma\left(\frac{1}{2}-k+m\right) \Gamma\left(\frac{1}{2}-k-m\right)} E\left(\rho, \frac{1}{2}-k+m, \frac{1}{2}-k-m:: z\right),
\end{aligned}
$$

where $R(\rho)>0$.

(15) in combination with (8) (with $n=1$ ) gives

$$
\begin{aligned}
& \int_{0}^{\infty} \exp \left(-\lambda-\frac{z}{2 \lambda}\right) \lambda^{\rho-l-1} W_{k_{1} m}\left(\frac{z}{\lambda}\right) d y \\
& =z^{-l} \times \sum_{i,-i} \frac{1}{i} E\left[\begin{array}{c}
\frac{1}{2}+m+l, \frac{1}{2}-m+l, 1, \rho: z e^{i \pi} \\
1-k+l
\end{array}\right]
\end{aligned}
$$


where $R(\rho)>0$.

Here apply (7) to the right hand side and get

$$
\begin{gathered}
\int_{0}^{\infty} \exp \left(-\lambda+\frac{z}{2 \lambda}\right) \lambda^{\rho+l-1} W_{k_{1} m}\left(\frac{z}{\lambda}\right) d \lambda=z^{-l}(2 \pi)^{1-n} n^{l+\rho-k} \\
\times \frac{1}{2 \pi} \sum_{i,-i} \frac{1}{i} E\left[\begin{array}{c}
\left.\Delta\left(n ; \frac{1}{2}+m+l\right), \Delta\left(n ; \frac{1}{2}-m+l\right), \Delta(n ; \rho):\left(\frac{z}{n^{2}}\right)^{n} e^{i \pi}\right] \\
\Delta(n ; 1-k+l)
\end{array}\right]
\end{gathered}
$$

where $n$ is any positive integer, $R(\rho)>0$.

When $l=k$, the last formula gives if $R(\rho)>0$ and $n$ is any positive integer

$$
\begin{gathered}
\int_{0}^{\infty} \exp \left(-\lambda-\frac{z}{2 \lambda}\right) \lambda^{\rho-k-1} W_{k_{1} m}\left(\frac{z}{\lambda}\right) d \lambda=2^{-k}(2 \pi)^{1-n} n \rho \\
\frac{1}{2 \pi} \sum_{i,-i} \frac{1}{i} E\left[\begin{array}{c}
\Delta\left(n ; \frac{1}{2}+m+k\right), \Delta\left(n ; \frac{1}{2}-m+k\right), \Delta(n, \rho):\left(\frac{z}{n^{2}}\right)^{n} e^{i \pi} \\
\frac{1}{n}, \frac{2}{n}, \ldots, \frac{n-1}{n} .
\end{array}\right]
\end{gathered}
$$

In (9) replace $\rho$ by $l+k, \lambda$ by $\frac{\lambda}{z}$, then replace $z$ by $1 /(1-z)$, take

$$
\alpha=\frac{1}{2}-k+m, \quad \beta=\frac{1}{2}-k-m,
$$

apply (1), so getting

$$
\begin{aligned}
& \int_{0}^{\infty} e^{-\lambda\left(\frac{1}{2}-z\right)} \lambda^{l-1} W_{k_{1} m}(\lambda) d \lambda=\frac{\pi}{\sin (l+k) \pi \Gamma\left(\frac{1}{2}-k+m\right) \Gamma\left(\frac{1}{2}-k-m\right)} \\
& \left\{\frac{\Gamma\left(\frac{1}{2}-k+m\right) \Gamma\left(\frac{1}{2}-k-m\right)}{\Gamma(1-l-k)}(1-z)^{-k-l} F\left[\begin{array}{c}
\frac{1}{2}-k+m, \frac{1}{2}-k-m ; 1-z \\
1-l-k
\end{array}\right]\right. \\
& \left.-\frac{\Gamma\left(\frac{1}{2}+m+l\right) \Gamma\left(\frac{1}{2}-m+l\right)}{\Gamma(1+l+k)} F\left[\begin{array}{c}
\frac{1}{2}+m+l, \frac{1}{2}-m+l ; 1-z \\
1+l+k
\end{array}\right]\right\}
\end{aligned}
$$

In virtue of the formula (p. 352.):

$$
E\left(P ; \alpha_{r}: q ; \rho_{s}: z\right)=\frac{\Gamma\left(\alpha_{1}\right) \ldots \Gamma\left(\alpha_{p}\right)}{\Gamma\left(\rho_{1}\right) \ldots \Gamma\left(\rho_{q}\right)^{p}} F_{q}\left(p ; \alpha_{r}: q ; \rho_{s} ;-\frac{1}{z}\right) .
$$

The expression between brachets \{\} in $A$ can be simplified by the formula ([1], p. 349)

$$
\begin{aligned}
& F(\alpha, \beta ; \gamma ; z)=\frac{\Gamma(\gamma-\alpha-\beta) \Gamma(\gamma)}{\Gamma(\gamma-\alpha) \Gamma(\gamma-\beta)} F(\alpha, \beta ; \alpha+\beta-\gamma+1 ; 1-z) \\
& +\frac{\Gamma(\alpha+\beta-\gamma) \Gamma(\gamma)}{\Gamma(\alpha) \Gamma(\beta)}(1-z)^{\gamma-\alpha-\beta} F(\gamma-\alpha, \gamma-\beta, \gamma-\alpha-\beta+1 ; 1-z)
\end{aligned}
$$


Thus A gives GOLDSTEIN'S, formula ([1], p. 396) namely

$$
\begin{aligned}
& \int_{0}^{\infty} e^{-\lambda\left(\frac{1}{2}-z\right)} \lambda^{l-1} W_{k_{1} m}(\lambda) d \lambda \\
= & \frac{\Gamma\left(l+m+\frac{1}{2}\right) \Gamma\left(l-m+\frac{1}{2}\right)}{\Gamma(l-k+1)} F\left(\begin{array}{c}
l+m+\frac{1}{2}, l-m+\frac{1}{2} ; z \\
l-k+1
\end{array}\right),
\end{aligned}
$$

where

$$
R(z)<1, R(l \pm m)>-\frac{1}{2}
$$

Also (9) (with $n=1$ ) in combination with (15) gives GOLDSTEIN'S formula (26) again.

(1) in combination with (9) gives if $R(\rho \pm n)>0$

$$
\begin{gathered}
\int_{0}^{\infty} e^{\frac{z^{2}}{2 \lambda^{2}}} \lambda^{\rho+2 k-1} K_{n}(2 \lambda) W_{k_{1} m}\left(\frac{z^{2}}{\lambda^{2}}\right) d \lambda=\frac{z^{2 k}}{4 \Gamma\left(\frac{1}{2}-k+m\right) \Gamma\left(\frac{1}{2}-k-m\right)} \\
E\left(\frac{1}{2}-k+m, \frac{1}{2}-k-m, \frac{1}{2} \rho+\frac{1}{2} n, \frac{1}{2} \rho-\frac{1}{2} n: z^{2}\right),
\end{gathered}
$$

(15) in combination with (9) gives if $R(\rho \pm n)>0$

$$
\begin{aligned}
& \int_{0}^{\infty} e^{\frac{z^{2}}{2 \lambda^{2}}} \lambda^{p-2 l-1} K_{n}(2 \lambda) W_{k_{1} m}\left(\frac{z^{2}}{\lambda^{2}}\right) d \lambda \\
= & \frac{1}{8 \pi} \sum_{i,-i} \frac{1}{i} E\left(\frac{1}{2}+m+l, \frac{1}{2}-m+l, 1, \frac{1}{2} \rho+\frac{1}{2} n, \frac{1}{2} \rho-\frac{1}{2} n: 1-k+l: e^{i \pi} z^{2}\right)
\end{aligned}
$$

When $k=l,(28)$ gives if $R(\rho \pm n)>0$

$$
\begin{aligned}
& \int_{0}^{\infty} e^{-\frac{z^{2}}{2 \lambda^{2}}} \lambda^{\rho-2 k-1} K_{n}(2 \lambda) W_{k_{1} m}\left(\frac{z^{2}}{\lambda^{2}}\right) d \lambda \\
= & \frac{z^{-2 k}}{8 \pi} \sum_{i,-i} \frac{1}{i} E\left(\frac{1}{2}+m+k, \frac{1}{2}-m+k, \frac{1}{2} \rho-\frac{1}{2} n, \frac{1}{2} \rho+\frac{1}{2} n: e^{i \pi} z^{2}\right) .
\end{aligned}
$$

(1) in combination with (10) gives

$$
\begin{aligned}
& 4 i \pi \int_{0}^{\infty} \exp \left(\frac{z^{2}}{2 \lambda^{2}}\right) \lambda^{\rho+2 k-1} J_{n}(2 \lambda) W_{k_{1} m}\left(z^{2} / \lambda^{2}\right) d \lambda= \\
& \frac{z^{2 k}}{\Gamma\left(\frac{1}{2}-k+m\right) \Gamma\left(\frac{1}{2}-k-m\right)} i^{\rho-n} E\left(\frac{1}{2}-k+m, \frac{1}{2}-k-m, \frac{1}{2} \rho+\frac{1}{2} n, \frac{1}{2} \rho-\frac{1}{2} n: z^{2} e^{-i \pi}\right) \\
& -\frac{z^{2 k}}{\Gamma\left(\frac{1}{2}-k+m\right) \Gamma\left(\frac{1}{2}-k-m\right)} i^{n-\rho} E\left(\frac{1}{2}-k+m, \frac{1}{2}-k-m, \frac{1}{2} \rho+\frac{1}{2} n, \frac{1}{2} \rho-\frac{1}{2} n: z^{2} e^{i \pi}\right),
\end{aligned}
$$


where

$$
R(\rho+n)>0, R\left(\frac{5}{2}-2 k-\rho \pm 2 m\right)>0,|\operatorname{amp} z|<\pi
$$

(15) in combination with 10) gives

$$
\begin{aligned}
& 4 i \pi \int_{0}^{\infty} \exp \left(-\frac{z^{2}}{2 \lambda^{2}}\right) \lambda^{\rho-2 \rho-1} J_{n}(2 \lambda) W_{k_{1} m}\left(z^{2} / \lambda^{2}\right) d \lambda \\
& =z^{-\frac{-n}{2 \pi}} \sum_{i,-i} \frac{1}{i} E\left(\frac{1}{2}+m+l, \frac{1}{2}-m+l, 1, \frac{1}{2} \rho+\frac{1}{2} n, \frac{1}{2} \rho-\frac{1}{2} n: 1-k+l: z^{2} e^{-i \pi}\right) \\
& =-z^{-2 l} i^{n-\rho} \frac{1}{2 \pi} \sum_{i,-i} \frac{1}{i} E\left(\frac{1}{2}+m+l, \frac{1}{2}-m+l, 1, \frac{1}{2} \rho+\frac{1}{2} n, \frac{1}{2} \rho-\frac{1}{2} n: 1-k+l: z^{2} e^{i \pi}\right)
\end{aligned}
$$

where

$$
R(\rho+n)>0, R\left(\frac{5}{2}+2 l-\rho \pm 2 m\right)>0 .
$$

When $k=l$, (31) gives if

$$
\begin{gathered}
R(\rho+n)>0, R\left(\frac{5}{2}+2 k-\rho \pm 2 m\right)>0,|\operatorname{amp} z|<\pi \\
(4 i \pi) z^{2 l} \int_{0}^{\infty} \exp \left(-\frac{z^{2}}{\lambda^{2}}\right) \lambda^{\rho-2 k-1} J_{n}(2 \lambda) W_{k_{1} m}\left(z^{2} / \lambda^{2}\right) d \lambda \\
=i^{\rho-n} \times \frac{1}{2 \pi} \sum_{i,-i} \frac{1}{i} E\left(\frac{1}{2}+m+l, \frac{1}{2}-m+l, \frac{1}{2} \rho+\frac{1}{2} n, \frac{1}{2} \rho-\frac{1}{2} n: z^{2} e^{-i \pi}\right) \\
-i^{n-\rho} \times \frac{1}{2 \pi} \sum_{i,-i} \frac{1}{i} E\left(\frac{1}{2}+m+l, \frac{1}{2}-m+l, \frac{1}{2} \rho+\frac{1}{2} n, \frac{1}{2} \rho-\frac{1}{2} n: z^{2} e^{i \pi}\right)
\end{gathered}
$$

(1) in combination with (12) gives

$$
\begin{aligned}
& \int_{0}^{\infty} \exp \left(\frac{1}{2} z^{2} \lambda^{2}\right) \lambda^{\rho-2 k-1} K_{n}(2 \lambda) W_{k_{1} m}\left(z^{2} \lambda^{2}\right) d \lambda \\
= & \frac{\pi^{2} 2^{\rho-2} z^{2 k} \Gamma\left(\frac{\rho+n}{2}\right) \Gamma\left(\frac{\rho-n}{2}\right)}{1}{ }_{2} F_{2}\left(\begin{array}{c}
\frac{1}{2}-k+m, \frac{1}{2}-k-m ;-1 / 4 z^{2} \\
1-\frac{\rho+n}{2}, 1-\frac{\rho-n}{2}
\end{array}\right) \\
+ & \frac{z^{2 k}}{\Gamma\left(\frac{1}{2}-k+m\right) \Gamma\left(\frac{1}{2}-k-m\right)} \sum_{n,-n} \Gamma\left(\frac{1}{2}-k+m+\frac{\rho+n}{2}\right) \\
\times & \Gamma\left(\frac{1}{2}-k-m+\frac{\rho+n}{2}\right) \Gamma(-n) \Gamma\left(-\frac{\rho+n}{2}\right) \\
& \times z^{-(\rho+n)}{ }_{2} F_{2}\left(\frac{1}{2}-k+m+\frac{\rho+n}{2}, \frac{1}{2}-k m+\frac{\rho+n}{2} ;-1 / 4 z^{2}\right) \\
& \times \frac{\rho+n}{2}, 1+n
\end{aligned}
$$


where

$$
R(\rho+n+1-2 k \pm 2 m)>0,|\operatorname{amp} z|<\pi .
$$

(15) in combination with (12) gives

$\int_{0}^{\infty} \exp \left(-\frac{1}{2} z^{2} \lambda^{2}\right) \lambda^{\rho+2 l-1} W_{k_{1} m}\left(z^{2} \lambda^{2}\right) K_{n}(\lambda) d \lambda$

$=\pi \sum_{n \rightarrow-n}\left[\begin{array}{c}\frac{\Gamma(-n) \Gamma\left(\frac{1}{2}+m+l+\frac{1}{2} \rho+\frac{1}{2} n\right) \Gamma\left(\frac{1}{2}-m+l+\frac{1}{2} \rho+\frac{1}{2} n\right)}{\Gamma\left(1-k+l+\frac{1}{2} \rho+\frac{1}{2} n\right) z^{\rho+n}} \\ { }_{2} F_{2}\left(\frac{1}{2}+m+l+\frac{1}{2} \rho+\frac{1}{2} n, \frac{1}{2}-m+l+\frac{1}{2} \rho+\frac{1}{2} n ; 1+n, 1-k+l+\frac{1}{2} \rho+\frac{1}{2} n ;-\frac{1}{4 z^{2}}\right)\end{array}\right]$

where

$$
R(\rho \pm n \pm 2 m+2 \rho+1)>0, R(\rho \pm n+2)>0
$$

and $z$ is real and positive.

(1) in combination with (13) gives

$$
\begin{aligned}
& \Gamma\left(\frac{1}{2}-k+m\right) \Gamma\left(\frac{1}{2}-k-m\right) z^{-2 k} \int_{0}^{\infty} \exp \left(-\mu+\frac{z^{2}}{2 \mu^{2}}\right) \mu^{\rho+2 k-1} I_{n}(\mu) W_{k_{1} m}\left(\frac{z^{2}}{\mu^{2}}\right) d \mu \\
& =\frac{\sin (\rho-n) \pi}{(2 \sqrt{2}) \cos (\rho \pi)} E\left[\begin{array}{c}
\left.\frac{1}{2}-k+m, \frac{1}{2}-k+m, \frac{\rho+n}{2}, \frac{\rho-n}{2}, \frac{\rho+n+1}{2}, \frac{\rho-n+1}{2}: z^{2}\right] \\
\frac{3}{4}+\frac{1}{2} \rho, \frac{1}{4}+\frac{1}{2} \rho
\end{array}\right] \\
& -\frac{\cos (n \pi)}{(4 \sqrt{2} \cdot \pi) \sin \left(\frac{\rho}{2}-\frac{1}{4}\right) \pi} z^{\frac{\rho}{2}-\frac{1}{4}} \\
& E\left[\begin{array}{c}
\frac{1}{2}-k+m+\frac{1}{4}-\frac{\rho}{2}, \frac{1}{2}-k-m+\frac{1}{4}-\frac{\rho}{2}, \frac{1}{4}+\frac{n}{2}, \frac{3}{4}+\frac{n}{2}, \frac{1}{4}-\frac{n}{2}, \frac{3}{4}-\frac{n}{2}: z^{2} \\
\frac{5}{4}-\frac{\rho}{2}, \frac{1}{2}
\end{array}\right] \\
& -\frac{\cos (n \pi)}{(4 \sqrt{2} \cdot \pi) \sin \left(\frac{3}{4}-\frac{\rho}{2}\right) \pi} z^{\frac{\rho}{2}-\frac{3}{4}} \\
& E\left[\begin{array}{c}
\frac{1}{2}-k+m+\frac{3}{4}-\frac{\rho}{2}, \frac{1}{2}-k-m+\frac{3}{4}-\frac{\rho}{2}, \frac{3}{4}+\frac{n}{2}, \frac{5}{4}+\frac{n}{2}, \frac{3}{4}-\frac{n}{2}, \frac{5}{4}-\frac{n}{2}: z^{2} \\
\frac{7}{4}-\frac{\rho}{2}, \frac{3}{2}
\end{array}\right]
\end{aligned}
$$

where

$$
R(\rho+n)>0, R(1-2 k \pm 2 m-\rho)>-\frac{1}{2},|\operatorname{amp} z|<\pi
$$


(15) in combination with (13) gives

$$
\begin{aligned}
& z^{2 l} \int_{0}^{\infty} \exp \left(-\mu-\frac{z^{2}}{2 \mu^{2}}\right) \mu^{\rho-2 l-1} I_{n}(\mu) W_{k_{1}, m}\left(\frac{z^{2}}{\mu^{2}}\right) d \mu \\
& =\frac{1}{2 \pi} \cdot \frac{\sin (\rho-n) \pi}{2 \sqrt{2} \cdot \cos (\rho \pi)} \\
& \times \sum_{i,-i} \frac{1}{i} E\left[\begin{array}{c}
\frac{1}{2}+m+l, \frac{1}{2}-m+l, 1, \frac{1}{2} \rho+\frac{1}{2} n, \frac{1}{2} \rho+\frac{1}{2} n+\frac{1}{2} \\
\frac{1}{2} \rho-\frac{1}{2} n, \frac{1}{2} \rho-\frac{1}{2} n+\frac{1}{2}: e^{i \pi} z^{2} \\
\frac{3}{4}+\frac{1}{2} \rho, \frac{1}{4}+\frac{1}{2} \rho, 1-k+l
\end{array}\right] \\
& -\frac{\cos (n \pi)}{2 \pi(4 \sqrt{2} \cdot \pi) \sin \left(\frac{3}{4}-\frac{\rho}{2}\right) \pi} z^{\rho-\frac{1}{2}} \\
& \times \sum_{i,-i} \frac{1}{i} E\left[\begin{array}{c}
\frac{1}{2}+m+l+\frac{1}{4}-\frac{\rho}{2}, \frac{1}{2}-m+l+\frac{1}{4}-\frac{\rho}{2}, \frac{5}{4}+\frac{\rho}{2} \\
\frac{1}{4}+\frac{n}{2}, \frac{3}{4}+\frac{n}{2}, \frac{1}{4}-\frac{n}{2}, \frac{3}{4}-\frac{n}{2}: z^{2} e^{i \pi} \\
\frac{5}{4}-\frac{\rho}{2}, \frac{1}{2}, 1-k+l+\frac{1}{4}-\frac{\rho}{2}
\end{array}\right] \\
& -\frac{\cos (n \pi)}{2 \pi(4 \sqrt{2} \cdot \pi) \sin \left(\frac{3}{4}-\frac{\rho}{2}\right) \pi} z^{\rho-\frac{3}{2}} \\
& +\sum_{i,-i} \frac{1}{i} E\left[\begin{array}{c}
\frac{1}{2}+m+l+\frac{3}{4}-\frac{\rho}{2}, \frac{5}{4}-m+l-\frac{\rho}{2}, \frac{7}{4}+\frac{\rho}{2} \\
\frac{3}{4}+\frac{n}{2}, \frac{5}{4}+\frac{n}{2}, \frac{3}{4}-\frac{n}{2}, \frac{5}{4}-\frac{n}{2}: z^{2} e^{i \pi} \\
\frac{7}{4}-\frac{\rho}{2}, \frac{3}{2}, \frac{7}{4}-k+l-\frac{\rho}{2}
\end{array}\right]
\end{aligned}
$$

where

$$
R(\rho+n)>0, R(1+2 l \pm 2 m-\rho)>-\frac{1}{2}, R\left(\frac{5}{2}-\rho\right)>0,|\operatorname{amp} z|<\pi .
$$

(1) in combination with (14) gives

$$
\begin{aligned}
& \Gamma\left(\frac{1}{2}-k+m\right) \Gamma\left(\frac{1}{2}-k-m\right) z^{-2 k} \int_{0}^{\infty} \exp \left(-\mu+\frac{1}{2} z^{2} \mu^{2}\right) \mu^{\rho-2 k-1} I_{n}(\mu) W_{k_{1} m}\left(z^{2} \mu^{2}\right) d \mu \\
& =\frac{\pi \Gamma\left(\frac{1}{2}-k+m\right) \Gamma\left(\frac{1}{2}-k-m\right) \Gamma\left(\frac{1}{4}-\frac{\rho}{2}\right) \Gamma\left(\frac{3}{4}-\frac{\rho}{2}\right)}{(\sqrt{2}) \sin (\rho+n) \pi \Gamma\left(1+\frac{\rho+n}{2}\right) \Gamma\left(\frac{1}{2}\right) \Gamma(1+n) \Gamma\left(\frac{1}{2}+n\right)}
\end{aligned}
$$




$$
\begin{aligned}
& { }_{4} F_{4}\left(\begin{array}{c}
\frac{1}{2}-k+m, \frac{1}{2}-k-m, \frac{1}{4}-\frac{\rho}{2}, \frac{3}{4}-\frac{\rho}{2} ;-1 / z^{2} \\
1+\frac{\rho+n}{2}, \frac{1}{2}, 1+n, \frac{1}{2}+n
\end{array}\right) \\
& +\frac{\pi^{-\frac{1}{2}} \Gamma\left(\frac{1}{2}-k+m+\frac{\rho+n}{2}\right) \Gamma\left(\frac{1}{2}-k-m+\frac{\rho+n}{2}\right) \Gamma\left(\frac{1}{4}+\frac{n}{2}\right) \Gamma\left(\frac{3}{4}+\frac{n}{2}\right) \Gamma\left(-\frac{\rho+n}{2}\right)}{2 \sqrt{2} \Gamma(1+n) \Gamma\left(\frac{1}{2}+n\right) z^{\rho+n}} \\
& \times{ }_{4} F_{4}\left(\begin{array}{c}
\left.\frac{1}{2}-k+m+\frac{\rho+n}{2}, \frac{1}{2}-k-m+\frac{\rho+n}{2}, \frac{1}{4}+\frac{n}{2}, \frac{3}{4}+\frac{n}{2} ;-1 / z^{2}\right) \\
1+\frac{\rho+n}{2}, \frac{1}{2}, 1+n, \frac{1}{2}+n
\end{array}\right) \\
& +\frac{\Gamma\left(1-k+m+\frac{\rho+n}{2}\right) \Gamma\left(1-k-m+\frac{\rho+n}{2}\right), \Gamma\left(\frac{3}{4}+\frac{n}{2}\right) \Gamma\left(\frac{5}{4}+\frac{n}{2}\right) \Gamma\left(-\frac{\rho+n+1}{2}\right)}{2 \sqrt{2} \Gamma(3 / 2) \Gamma\left(\frac{3}{2}+n\right) \Gamma(1+n) z^{\rho+n+1}} \\
& \times{ }_{4} F_{4}\left(\begin{array}{c}
1-k+m+\frac{\rho+n}{2}, 1-k-m+\frac{\rho+n}{2}, \frac{3}{4}+\frac{n}{2}, \frac{5}{4}+\frac{n}{2} ;-1 / z^{2} \\
1+\frac{\rho+n+1}{2}, \frac{3}{2}, \frac{3}{2}+n, 1+n
\end{array}\right)
\end{aligned}
$$

where

$$
R(n+\rho+1-2 k \pm 2 m)>0,|\operatorname{amp} z|<\pi, R\left(\frac{1}{2}-\rho\right)>0
$$

(15) in combintation with (14) gives

$$
\begin{aligned}
& z^{2 l} \int_{0}^{\infty} \exp \left(-\mu-\frac{1}{2} z^{2} \mu^{2}\right) \mu^{\rho+2 l-1} I_{n}(\mu) W_{k_{1} m}\left(z^{2} \mu^{2}\right) d \mu \\
= & \frac{1}{2 \sqrt{2}} \frac{\Gamma\left(\frac{1}{2}+m+l+\frac{\rho+n}{2}\right) \Gamma\left(\frac{1}{2}-m+l+\frac{\rho+n}{2}\right) \Gamma\left(\frac{1}{4}+\frac{n}{2}\right) \Gamma\left(\frac{3}{4}+\frac{n}{2}\right)}{\Gamma\left(\frac{1}{2}\right) \Gamma\left(1-k+l+\frac{\rho+n}{2}\right) \Gamma(1+n) \Gamma\left(\frac{1}{2}+n\right)} z^{-(\rho+n)} \\
& \times{ }_{4} F_{4}\left(\frac{\left.\frac{1}{2}+m+l+\frac{\rho+n}{2}, \frac{1}{2}-m+l+\frac{\rho+n}{2}, \frac{1}{4}+\frac{n}{2}, \frac{3}{4}+\frac{n}{2} ; 1 / z^{2}\right)}{\Gamma\left(\frac{3}{2}\right) \Gamma\left(1-k+\rho+\frac{1}{2}+\frac{\rho+n+1}{2}, 1+n, \frac{\rho}{2}+n\right.}\right) \\
& +\frac{1}{2 \sqrt{2}} \frac{\Gamma\left(1+m+l+\frac{\rho}{2}+n\right) \Gamma(1+n)}{\frac{\rho}{2}+n\left(1-m+l+\frac{\rho+n}{2}\right) \Gamma\left(\frac{3}{4}+\frac{n}{2}\right) \Gamma\left(\frac{5}{4}+\frac{n}{2}\right) z^{-(\rho+n+1)}} \\
& \times{ }_{4} F_{4}\left(\begin{array}{c}
1+m+l+\frac{\rho+n}{2}, 1-m+l+\frac{\rho+n}{2}, \frac{3}{4}+\frac{n}{2}, \frac{5}{4}+\frac{n}{2} ; 1 / z^{2} \\
\frac{3}{2}-k+\rho+\frac{\rho+n}{2}, \frac{3}{2}+n, 1+n
\end{array}\right)
\end{aligned}
$$


where

$$
R(n+p+1 \pm 2 m+2 l)>0, R\left(\frac{1}{2}-p\right)>0, \text { and }|\operatorname{amp} z|<\pi .
$$

It may be noted that the formula (22), (29), (31), (32), (36) and (38) can be generalized by applying (7).

\section{$\S$ 4. Integerals involving the product of two Whittaker functions:}

(4) in combination with (8) $(n=2)$ gives if $R(\rho)>0$

$$
\begin{aligned}
\int_{0}^{\infty} & e^{-\lambda} \lambda^{\rho+2 k-1} W_{k_{1} m}\left(2 i \frac{z}{\lambda}\right) W_{k_{1} m}\left(-2 i \frac{z}{\lambda}\right) d \lambda=\frac{2^{\rho-1} z^{2 k}}{\pi \Gamma\left(\frac{1}{2}-k+m\right) \Gamma\left(\frac{1}{2}-k-m\right)} \\
& \times E\left[\frac{1}{2}-k+m, \frac{1}{2}-k-m, \frac{1}{2}-k, 1-k, \frac{1}{2} \rho, \frac{1}{2} \rho+\frac{1}{2}: 1-2 k: z^{2} / 4\right]
\end{aligned}
$$

(4) in combination with (9) (with $n=2$ ) gives

$$
\begin{aligned}
& \int_{0}^{\infty} e^{-\lambda} \lambda^{\rho-2 k-1} W_{k_{1} m}(2 i z \lambda) W_{k_{1}, m}(-2 i z \lambda) d \lambda \\
= & \frac{2 \rho \pi^{\frac{3}{2}} \operatorname{cosec}(\rho \pi) \cdot z^{2 k}}{2^{-2 k} \Gamma\left(1-\frac{\rho}{2}\right) \Gamma\left(\frac{1}{2}-\frac{\rho}{2}\right)}{ }_{4} F_{3}\left(\begin{array}{c}
\left.\frac{1}{2}-k+m, \frac{1}{2}-k-m, \frac{1}{2}-k, 1-k ;-\frac{1}{4 z^{2}}\right) \\
1-\frac{1}{2} \rho, \frac{1}{2}-\frac{1}{2} \rho, 1-2 k
\end{array}\right) \\
& -\frac{\pi^{\frac{1}{2}} z^{2 k-\rho} \Gamma\left(\frac{1}{2}-k+m+\frac{\rho}{2}\right) \Gamma\left(\frac{1}{2}-k-m+\frac{\rho}{2}\right) \Gamma\left(1-k+\frac{\rho}{2}\right) \Gamma\left(\frac{1}{2}-k+\frac{\rho}{2}\right)}{2 \Gamma\left(\frac{1}{2}-k+m\right) \Gamma\left(\frac{1}{2}-k-m\right) \Gamma\left(1+\frac{\rho}{2}\right) \Gamma\left(1-2 k+\frac{\rho}{2}\right) \sin \left(\frac{\rho}{2}\right) \pi} \\
& \times{ }_{4} F_{3}\left(\frac{1}{2}-k+m+\frac{\rho}{2}, \frac{1}{2}-k-m+\frac{\rho}{2}, 1-k+\frac{\rho}{2}, \frac{1}{2}-k+\frac{\rho}{2} ; \frac{1}{2}, 1+\frac{\rho}{2}, 1-2 k+\frac{\rho}{2} ;-\frac{1}{4 z^{2}}\right) \\
& +\frac{2^{-2} \pi z^{2 k-\rho-1} \Gamma\left(\frac{1}{2}-k+m+\frac{\rho}{2}+\frac{1}{2}\right) \Gamma\left(1-k-m+\frac{\rho}{2}\right) \Gamma\left(\frac{3}{2}-k+\frac{\rho}{2}\right) \Gamma\left(1-k+\frac{\rho}{2}\right)}{\sin \left(\frac{\rho+1}{2}\right) \pi \Gamma\left(\frac{1}{2}-k+m\right) \Gamma\left(\frac{1}{2}-k-m\right) \Gamma\left(\frac{3}{2}+\frac{\rho}{2}\right) \Gamma\left(\frac{3}{2}\right) \Gamma\left(\frac{3}{2}-2 k+\frac{\rho}{2}\right)} \\
& \times{ }_{4} F_{3}\left(1-k+m+\frac{\rho}{2}, 1-k-m+\frac{\rho}{2}, \frac{3}{2}-k+\frac{\rho}{2}, 1-k+\frac{\rho}{2} ; \frac{3}{2}, \frac{3}{2}+\frac{\rho}{2}, \frac{3}{2}-2 k+\frac{\rho}{2} ;-\frac{1}{4 z^{2}}\right)
\end{aligned}
$$

where

$$
R(\rho+1-2 k \pm 2 m)>0,|\operatorname{amp} z|<\pi .
$$

(4) in combination with (10) gives if $R(\rho \pm n)>0$

$$
4 \int_{0}^{\infty} \lambda^{\rho-2 k-1} K_{n}(2 \lambda) W_{k_{1} m}(2 i z / \lambda) W_{k_{1} m}(-2 i z / \lambda) d \lambda
$$




$$
\begin{aligned}
= & \frac{z^{2 k}}{\Gamma\left(\frac{1}{2}\right) \Gamma\left(\frac{1}{2}-k+m\right) \Gamma\left(\frac{1}{2}-k-m\right)} \\
& E\left[\frac{1}{2}-k+m, \frac{1}{2}-k-m, \frac{1}{2}-k, 1-k, \frac{\rho+n}{2}, \frac{\rho-n}{2}: z^{2}\right]
\end{aligned}
$$

(4) in combination with (11) gives

$$
\begin{aligned}
& 4 i \pi \int_{0}^{\infty} \lambda^{\rho+2 k-1} J_{n}(2 \lambda) W_{k_{1} m}(2 i z / \lambda) W_{k_{1} m}(-2 i z / \lambda) d \lambda \\
= & \frac{z^{2 k} \cdot i^{n-\rho}}{\Gamma\left(\frac{1}{2}\right) \Gamma\left(\frac{1}{2}-k+m\right) \Gamma\left(\frac{1}{2}-k-m\right)} \\
& E \cdot\left[\frac{1}{2}-k+m, \frac{1}{2}-k-m, \frac{1}{2}-k, 1-k, \frac{\rho+n}{2}, \frac{\rho-n}{2}: z^{2} e^{i \pi}\right] \\
- & \frac{z^{2 k} i^{n-\rho}}{\Gamma\left(\frac{1}{2}\right) \Gamma\left(\frac{1}{2}-k+m\right) \Gamma\left(\frac{1}{2}-k-m\right)} \\
& E\left[\frac{1}{2}-k+m, \frac{1}{2}-k-m, \frac{1}{2}-k, 1-k, \frac{\rho+n}{2}, \frac{\rho-n}{2}: z^{2} e^{-i \pi}\right]
\end{aligned}
$$

where

$$
R(\rho+n)>0, R\left(\frac{3}{2}-\rho+1-2 k \pm 2 m\right)>0 R\left(\frac{5}{2}-\rho-2 \dot{k}\right)>0 .
$$

(4) in combination with (12) gives

$$
\begin{aligned}
& z^{-2 k} \sqrt{\pi} \Gamma\left(\frac{1}{2}-k+m\right) \Gamma\left(\frac{1}{2}-k-m\right) \int_{0}^{\infty} \lambda^{\rho-2 k-1} K_{n}(\lambda) W_{k_{1} m}(2 i z \lambda) W_{k_{1} m}(-2 i z / \lambda) d \lambda \\
= & \frac{2^{\rho-2} \Gamma\left(\frac{\rho+n}{2}\right) \Gamma\left(\frac{\rho-n}{2}\right) \Gamma\left(\frac{1}{2}-k+m\right) \Gamma\left(\frac{1}{2}-k-m\right) \Gamma\left(\frac{1}{2}\right)}{2^{-2 k}} \\
& { }_{4} F_{3}\left(\frac{\left.\frac{1}{2}-k+m, \frac{1}{2}-k-m, \frac{1}{2}-k, 1-k ;-\frac{1}{4 z^{2}}\right)}{1-\frac{\rho+n}{2}, 1-\frac{\rho-n}{2}, 1-2 k}\right) \\
+ & \sum_{n,-n} \frac{\Gamma(-n) \Gamma\left(\frac{1}{2}-k+m+\frac{\rho+n}{2}\right) \Gamma\left(\frac{1}{2}-k-m+\frac{\rho+n}{2}\right) \Gamma\left(\frac{1}{2}-k+\frac{\rho+n}{2}\right) \Gamma\left(1-k+\frac{\rho+n}{2}\right)}{\Gamma\left(1-2 k+\frac{\rho+n}{2}\right) z^{\rho+n}}
\end{aligned}
$$


${ }_{4} F_{3}\left(\begin{array}{c}\frac{1}{2}-k+m+\frac{\rho+n}{2}, \frac{1}{2}-k-m+\frac{\rho+n}{2}, \frac{1}{2}-k+\frac{\rho+n}{2}, 1-k+\frac{\rho+n}{2} ;-\frac{1}{4 z^{2}} \\ 1+\frac{\rho+n}{2}, 1+n, 1-2 k+\frac{\rho+n}{2}\end{array}\right)$

where

$$
R(\rho \pm n+1-2 k+2 m)>0, R(\rho \pm n+1-2 k)>0, \mid \text { amp } z \mid<\pi
$$

(4) in combination with (13) gives

$$
\begin{aligned}
& \int_{0}^{\infty} e^{-\mu} \mu^{\rho+2 k-1} I_{n}(\mu) W_{k_{1} m}\left(\frac{2 i z}{\mu}\right) W_{k_{1} m}\left(-\frac{2 i z}{\mu}\right) d \mu \\
& =\frac{z^{2 k} \sin (\rho-n) \pi}{\Gamma\left(\frac{1}{2}\right) \Gamma\left(\frac{1}{2}-k+m\right) \Gamma\left(\frac{1}{2}-k-m\right) \cdot(2 \sqrt{2}) \cos (\rho \pi)} \\
& \times E\left[\begin{array}{c}
\frac{1}{2}-k+m, \frac{1}{2}-k-m, \frac{1}{2}-k, 1-k, \frac{\rho+n}{2}, \frac{\rho-n}{2}, \frac{\rho+n+1}{2} \\
\frac{\rho-n+1}{2}: z^{2} \\
\frac{3}{4}+\frac{1}{2} \rho, \frac{1}{4}+\frac{1}{2} \rho, 1-2 k
\end{array}\right] \\
& -\frac{z^{2 k} \cos (n \pi) z^{\frac{\rho}{2}-\frac{1}{4}}}{\Gamma\left(\frac{1}{2}\right) \Gamma\left(\frac{1}{2}-k+m\right) \Gamma\left(\frac{1}{2}-k-m\right)(4 \sqrt{2} \pi) \sin \left(\frac{\rho}{2}-\frac{1}{4}\right) \pi} \\
& \times E\left[\begin{array}{c}
\frac{3}{4}-k+m-\frac{\rho}{2}, \frac{3}{4}-k-m-\frac{\rho}{2}, \frac{3}{4}-k-\frac{\rho}{2}, \frac{5}{4}-k-\frac{\rho}{2} \\
\frac{1}{4}+\frac{n}{2}, \frac{3}{4}+\frac{n}{2}, \frac{1}{4}-\frac{n}{2}, \frac{3}{4}-\frac{n}{2}: z^{2} \\
\frac{5}{4}-\frac{\rho}{2}, \frac{1}{2}, 1-2 k+\frac{1}{4}-\frac{\rho}{2}
\end{array}\right] \\
& -\frac{z^{2 k} \cos (n \pi) z^{\frac{\rho}{2}-\frac{3}{4}}}{\Gamma\left(\frac{1}{2}\right) \Gamma\left(\frac{1}{2}-k+m\right) \Gamma\left(\frac{1}{2}-k-m\right)(4 \sqrt{2} \pi) \sin \left(\frac{\rho}{2}-\frac{1}{4}\right) \pi} \\
& \times E\left[\begin{array}{c}
\frac{5}{4}-k+m+\frac{\rho}{2}, \frac{5}{4}-k-m-\frac{\rho}{2}, \frac{5}{4}-k-\frac{\rho}{2}, \frac{7}{4}-k-\frac{\rho}{2}, \\
\frac{3}{4}+\frac{n}{2}, \frac{5}{4}+\frac{n}{2}, \frac{3}{4}-\frac{n}{2}, \frac{5}{4}-\frac{n}{2}: z^{2} \\
\frac{7}{4}-\frac{\rho}{2}, \frac{3}{2}, \frac{7}{4}-2 k-\frac{\rho}{2}
\end{array}\right]
\end{aligned}
$$


where

$R(\rho+n)>0, R\left(1-2 k \pm 2 m-\rho+\frac{1}{2}\right)>0, R\left(\frac{3}{2}-2 k\right)>0,|\operatorname{amp} z|<\pi$

(4) in combination with (14) gives

$$
\begin{aligned}
& \int_{0}^{\infty} e^{-\mu} \mu^{\rho-2 k-1} I_{n}(\mu) W_{k_{1} m}(-2 i z \mu) W_{k_{1} m}(-2 i z \mu) d \mu \\
& =\frac{\sqrt{\pi} z^{2 k} \cdot \sqrt{\pi} \Gamma\left(\frac{1}{4}-\frac{\rho}{2}\right) \Gamma\left(\frac{3}{4}-\frac{\rho}{2}\right)}{\sqrt{2} \sin (\rho+n) \pi 2^{-2 k} \Gamma\left(1-\frac{\rho+n}{2}\right) \Gamma\left(\frac{1}{2}-\frac{\rho+n}{2}\right) \Gamma\left(1+\frac{n-\rho}{2}\right) \Gamma\left(\frac{1}{2}+\frac{n-\rho}{2}\right)} \\
& \times \frac{1}{\Gamma(1-k)}{ }_{0} F_{5}\left(\begin{array}{c}
\frac{1}{2}-k+m, \frac{1}{2}-k-m, \frac{1}{4}-\frac{\rho}{2}, \frac{3}{4}-\frac{\rho}{2}, 1-k, \frac{1}{2}-k ;-1 / z^{2} \\
1-\frac{\rho+n}{2}, \frac{1}{2}-\frac{\rho+n}{2}, 1+\frac{n-\rho}{2}, \frac{1}{2}+\frac{n-\rho}{2}, 1-2 k
\end{array}\right) . \\
& -\frac{z^{2 k} \sqrt{\pi} \Gamma\left(\frac{1}{2}-k+m+\frac{\rho+n}{2}\right) \Gamma\left(\frac{1}{2}-k-m+\frac{\rho+n}{2}\right) \Gamma\left(1-k+\frac{\rho+n}{2}\right)}{z^{\rho+n} 2 \sqrt{2} \cdot \sin \left(\frac{\rho+n}{2} \pi\right) \Gamma\left(\frac{1}{2}-k+m\right) \Gamma\left(\frac{1}{2}-k-m\right) \Gamma\left(1+\frac{\rho+n}{2}\right)} \\
& \times \frac{\Gamma\left(\frac{1}{2}-k+\frac{\rho+n}{2}\right) \Gamma\left(\frac{1}{4}+\frac{n}{2}\right) \Gamma\left(\frac{3}{4}+\frac{n}{2}\right)}{\Gamma\left(\frac{1}{2}\right) \Gamma\left(1-2 k+\frac{\rho+2}{2}\right) \Gamma(1+n) \Gamma\left(\frac{1}{2}+n\right)} \\
& \times{ }_{8} F_{5}\left(\begin{array}{c}
\frac{1}{2}-k+m+\frac{\rho+n}{2}, \frac{1}{2}-k-m+\frac{\rho+n}{2}, 1-k+\frac{\rho+n}{2} \\
\frac{1}{2}-k+\frac{\rho+n}{2}, \frac{1}{4}+\frac{n}{2}, \frac{3}{4}+\frac{n}{2} ;-\frac{1}{z^{2}} \\
1+\frac{\rho+n}{2}, \frac{1}{2}, 1-2 k+\frac{\rho+n}{2}, 1+n, \frac{1}{2}+n
\end{array}\right) \\
& -\frac{\sqrt{\pi} z^{2 k} \Gamma\left(1-k+m+\frac{\rho+n}{2}\right) \Gamma\left(1-k-m+\frac{\rho+n}{2}\right)}{z^{\rho+n+1}(2 \sqrt{2}) \cdot \cos \left(\frac{\rho+n}{2}\right) \pi \Gamma\left(\frac{1}{2}-k+m\right) \Gamma\left(\frac{1}{2}-k-m\right)} \\
& \times \frac{\Gamma\left(1-k+\frac{\rho+n}{2}\right) \Gamma\left(\frac{3}{2}-k+\frac{\rho+n}{2}\right) \Gamma\left(\frac{3}{4}+\frac{n}{2}\right) \Gamma\left(\frac{5}{4}+\frac{n}{2}\right)}{\Gamma\left(\frac{3}{2}+\frac{\rho+n}{2}\right) \Gamma\left(\frac{3}{2}\right) \Gamma\left(\frac{3}{2}-2 k+\frac{\rho+n}{2}\right) \Gamma\left(\frac{3}{2}+n\right) \Gamma(1+n)}
\end{aligned}
$$




$$
\times{ }_{8} F_{5}\left(\begin{array}{c}
1-k+m+\frac{\rho+n}{2}, 1-k-m+\frac{\rho+n}{2}, 1-k+\frac{\rho+n}{2}, \\
\frac{3}{2}-k+\frac{\rho+n}{2}, \frac{3}{4}+\frac{n}{2}, \frac{5}{4}+\frac{n}{2} ; \frac{1}{z^{2}} \\
\frac{3}{2}+\frac{\rho+n}{2}, \frac{3}{2}, \frac{3}{2}-2 k+\frac{\rho+n}{2}, \frac{3}{2}+n, 1+n
\end{array}\right)
$$

where

$$
\begin{gathered}
R(n+\rho+1-2 k \pm 2 m)>0, R(n+\rho+1-2 k)>0 \\
R\left(\frac{1}{2}-\rho\right)>0,|\operatorname{amp} z|<\pi .
\end{gathered}
$$

(19) in combination with 8 (with $n=2$ ) gives

$$
\begin{array}{r}
\int_{0}^{\infty} e^{-\lambda} \lambda^{\rho-l-1} W_{k_{1} m}(2 z / \lambda) W_{-k_{1} m}(2 z / \lambda) d \lambda=z^{-l} 2^{\rho-2} \pi^{-2} \sum_{i,-i} \frac{1}{i} \\
E\left[\begin{array}{c}
\left.\frac{1}{2}+\frac{l}{2}, 1+\frac{l}{2}, 1, \frac{l}{2}+m+\frac{1}{2}, \frac{l}{2}-m+\frac{1}{2}, \frac{\rho}{2}, \frac{\rho+1}{2}: e^{i \pi} \frac{z^{2}}{4}\right] \\
1+k+\frac{1}{2} l, 1+k-\frac{1}{2} l
\end{array}\right]
\end{array}
$$

where $R(\rho)>0$.

Here apply (7) and get,

$$
\begin{gathered}
\int_{0}^{\infty} e^{-\lambda} \lambda^{\rho-l-1} W_{k_{1} m}\left(\frac{2 z}{\lambda}\right) W_{-k_{1} m}\left(\frac{2 z}{\lambda}\right) d \lambda=2^{-2 l} 2^{2 \rho+2 l-2 k-4} \pi^{-4} z^{-1} \\
\times \sum_{i,-i} \frac{1}{i} E\left[\begin{array}{c}
\Delta\left(n ; \frac{1+l}{k}\right), \Delta\left(n ; 1+\frac{l}{2}\right), \Delta(n ; 1), \Delta\left(n ; \frac{l+1}{2}+m\right), \\
\Delta\left(n ; \frac{l-1}{2}+m\right), \Delta\left(n ; \frac{\rho}{2}\right), \Delta\left(n ; \frac{\rho+1}{2}\right):\left(\frac{z^{2}}{n^{4}}\right)^{n} e^{i \pi} \\
\Delta\left(n ; 1+k+\frac{1}{2} l\right), \Delta\left(n ; 1+k-\frac{1}{2} l\right)
\end{array}\right]
\end{gathered}
$$

where $n$ is any positive integer and $R(\rho)>0$.

(19) in combination with (9) gives

$$
\begin{aligned}
& \int_{0}^{\infty} e^{-\lambda} \lambda^{\rho+l-1} W_{k_{1} m}(2 z \lambda) W_{-k_{1}, m}(2 z \lambda) d \lambda \\
&= \frac{\Gamma\left(\frac{1}{2}+\frac{1}{2} l+\frac{1}{2} \rho\right) \Gamma\left(1+\frac{1}{2} l+\frac{1}{2} \rho\right) \Gamma\left(\frac{1}{2}+m+\frac{1}{2} l+\frac{1}{2} \rho\right) \Gamma\left(\frac{1}{2}-m+\frac{1}{2} l+\frac{1}{2} \rho\right)}{2 z^{\rho+1} \Gamma\left(\frac{1}{2}\right) \Gamma\left(1+k+\frac{1}{2} l+\frac{1}{2} \rho\right) \Gamma\left(1+k-\frac{1}{2} l+\frac{1}{2} \rho\right)} \\
& \times{ }_{4} F_{3}\left(\frac{1}{2}+\frac{1}{2} l+\frac{1}{2} \rho, 1+\frac{1}{2} l+\rho, \frac{1}{2}+m+\frac{1}{2} l+\frac{1}{2} \rho, \frac{1}{2}-m+\frac{1}{2} l+\frac{1}{2} \rho ; 1 / 4 z^{2}\right) \\
& \frac{1}{2}, 1+k+\frac{1}{2} l+\rho, 1+k-\frac{1}{2} l+\frac{1}{2} \rho
\end{aligned}
$$




$$
\begin{gathered}
-\frac{\Gamma\left(1+\frac{1}{2} l+\frac{1}{2} \rho\right) \Gamma\left(\frac{3}{2}+\frac{1}{2} l+\frac{1}{2} \rho\right) \Gamma\left(1+\frac{1}{2} l+m+\frac{1}{2} \rho\right) \Gamma\left(1+\frac{1}{2} l-m+\frac{1}{2} \rho\right)}{2^{2} z^{\rho+l+1} \Gamma\left(\frac{3}{2}\right) \Gamma\left(\frac{3}{2}+k+\frac{1}{2} l+\frac{1}{2} \rho\right) \Gamma\left(\frac{3}{2}+k-\frac{1}{2} l+\frac{1}{2} \rho\right)} \\
\times{ }_{4} F_{3}\left(\begin{array}{c}
1+\frac{1}{2} l+\frac{1}{2} \rho, \frac{3}{2}+\frac{1}{2} l+\frac{1}{2} \rho, 1+\frac{1}{2} l+m+\frac{1}{2} \rho, 1-m+\frac{1}{2} l+\frac{1}{2} \rho ; 1 / 4 z^{2} \\
\frac{3}{2}, \frac{3}{2}+k+\frac{1}{2} l+\frac{1}{2} \rho, \frac{3}{2}+k-\frac{1}{2} l+\frac{1}{2} \rho
\end{array}\right)
\end{gathered}
$$

where

$$
R(1+l+\rho)>0, R(2+\rho)>0, R(l \pm 2 m+1)>0 \text { and } z
$$

is real and positive.

(19) in combination with (10) gives if $R(\rho \pm n)>0$.

$$
\begin{gathered}
4 z^{l} \int_{0}^{\infty} \lambda^{\rho-l-1} W_{k_{1} m}\left(\frac{2 z}{\lambda}\right) W_{-k_{1} m}\left(\frac{2 \lambda}{\lambda}\right) K_{n}(2 \lambda) d \lambda \\
=\pi^{-\frac{1}{2}} \times \frac{1}{2 \pi} \sum_{i,-i} \frac{1}{i} E\left[\begin{array}{c}
\frac{1}{2}+\frac{\rho}{2}, 1+\frac{l}{2}, 1, \frac{l}{2}+m+\frac{1}{2}, \frac{l}{2}-m+\frac{1}{2}, \frac{\rho+n}{2}, \frac{\rho-n}{2} e^{i \pi} z^{2} \\
1+k+\frac{1}{2} l, 1+k+l
\end{array}\right]
\end{gathered}
$$

(19) in combination with (11) gives

$$
\begin{aligned}
& 4 i \pi z^{l} \int_{0}^{\infty} \lambda^{\rho-2 l-1} J_{n}(2 \lambda) W_{-k_{1} m}\left(\frac{2 z}{\lambda}\right) W_{k_{1} m}\left(\frac{2 z}{\lambda}\right) d \lambda \\
& =\pi^{-3 / 2} \sin \left(\frac{\rho-n}{2}\right) \pi \\
& \times E\left[\begin{array}{c}
\frac{1}{2}+\frac{l}{2}, 1+\frac{l}{2}, 1, \frac{l}{2}+m+\frac{1}{2}, \frac{l}{2}-m+\frac{1}{2}, \frac{\rho+n}{2}, \frac{\rho-n}{2}: z^{2} \\
1+k+\frac{1}{2} l, 1+k-\frac{1}{2} l
\end{array}\right] \\
& +\frac{1}{2 \pi^{3 / 2}} i^{\rho-n-1} \\
& \times E\left[\begin{array}{c}
\frac{1}{2}+\frac{l}{2}, 1+\frac{1}{2} l, 1, \frac{l}{2}+m+\frac{1}{2}, \frac{l}{2}-m+\frac{1}{2}, \frac{\rho+n}{2}, \frac{\rho-n}{2}: z^{2} e^{-2 i \pi} \\
1+k+\frac{1}{2} l, 1+k-\frac{1}{2} l
\end{array}\right] \\
& -\frac{1}{2 \pi^{3 / 2}} i^{n-\rho-1} \\
& \times E\left[\begin{array}{c}
\frac{1}{2}+\frac{l}{2}, 1+\frac{l}{2}, 1, \frac{l}{2}+m+\frac{1}{2}, \frac{l}{2}-m+\frac{1}{2}, \frac{\rho+n}{2}, \frac{\rho-n}{2}: z^{2} e^{2 i \pi} \\
1+k+\frac{1}{2} l, 1+k-\frac{1}{2} l
\end{array}\right]
\end{aligned}
$$


where

$$
\begin{gathered}
R(\rho+n)>0, R\left(\frac{3}{2}-\rho+1+l\right)>0, R\left(\frac{7}{2}-\rho\right)>0 \\
R\left(\frac{3}{2}-\rho+1 \pm 2 m+l\right)>0 \text { and } z
\end{gathered}
$$

is real and positive.

(19) in combination with (12) gives

$$
\begin{aligned}
& \int_{0}^{\infty} \lambda^{p+l-1} K_{n}(\lambda) W_{k_{1} m}(2 z \lambda) W_{-k_{1} m}(2 z \lambda) d \lambda= \\
& \sum_{n,-n} \frac{\Gamma\left(\frac{l+\rho+n+1}{2}\right) \Gamma\left(1+\frac{\rho+l+n}{2}\right) \Gamma\left(m+\frac{l+\rho+n+1}{2}\right) \Gamma\left(-m+\frac{l+\rho+n+1}{2}\right) \Gamma(-n)}{z^{\rho+n+l} \Gamma\left(1+k+\frac{l+\rho+n}{2}\right) \Gamma\left(1+k+\frac{\rho+n-l}{2}\right) \Gamma\left(\frac{1}{2}\right)} \\
& \times{ }_{4} F_{3}\left(\begin{array}{c}
\frac{1}{2}+\frac{\rho}{2}+\frac{\rho}{2}+\frac{n}{2}, 1+\frac{l}{2}+\frac{\rho}{2}+\frac{n}{2}, m+\frac{l+\rho+n+1}{2},-m+\frac{l+\rho+n+1}{2} ; 1 / 4 z^{2} \\
1+n, 1+k+\frac{l+\rho+n}{2}, 1+k+\frac{\rho+n-l}{2}
\end{array}\right)
\end{aligned}
$$

where $z$ is real and positive and $\left|4 z^{2}\right|>1$.

(13) in combination with (19) gives

$$
\begin{aligned}
& \int_{0}^{\infty} e^{-\mu} \mu^{\rho-l-1} I_{n}(\mu) W_{k_{1} m}\left(\frac{2 z}{\mu}\right) W_{-k_{1} m}\left(\frac{2 z}{\mu}\right) d \mu=\frac{\sin (\rho-n) \pi z^{-l}}{\sqrt{\pi}(2 \sqrt{2}) \cos (\rho \pi)(2 \pi)} \\
& \times \sum_{i,-i} \frac{1}{i} E\left[\begin{array}{c}
\frac{l+1}{2}, 1+\frac{l}{2}, 1, \frac{l+1}{2}+m, \frac{l+1}{2}-m, \frac{\rho+n}{2}, \frac{\rho+n+1}{2}, \\
\frac{\rho-n}{2}, \frac{\rho-n+1}{2}: e^{i \pi} z^{2} \\
\frac{3}{4}+\frac{l}{2}, \frac{1}{4}+\frac{l}{2}, 1+k+\frac{1}{2} l, 1+k-\frac{1}{2} l
\end{array}\right] \\
& -\frac{\pi^{-\frac{1}{2}} \cos (n \pi) z^{\rho-\frac{1}{2}-l}}{(\sqrt{2} \cdot \pi) \sin \left(\frac{\rho}{2}-\frac{1}{4}\right) \pi(2 \pi)} \\
& \times \sum_{i,-i} \frac{1}{i} E\left[\begin{array}{c}
\frac{3}{4}+\frac{l}{2}-\frac{\rho}{2}, \frac{5}{4}+\frac{l}{2}-\frac{\rho}{2}, \frac{3}{4}+m+\frac{l-\rho}{2}, \frac{3}{4}-m+\frac{l-\rho}{2} \\
\frac{1}{4}+\frac{n}{2}, \frac{3}{4}+\frac{n}{2}, \frac{1}{4}-\frac{n}{2}, \frac{3}{4}-\frac{n}{2}: e^{i \pi} z^{2} \\
\frac{1}{2}, \frac{5}{4}+k+\frac{l-\rho}{2}, \frac{5}{4}+k-\frac{l+\rho}{2}
\end{array}\right] \\
& -\frac{\pi^{\frac{1}{2}} \cos (n \pi) z^{\rho-\frac{3}{2}-l}}{(4 \sqrt{2} \cdot \pi) \sin \left(\frac{3}{4}-\frac{\rho}{2}\right) \pi(2 \pi)}
\end{aligned}
$$




$$
\times \sum_{i,-i} \frac{1}{i} E\left[\begin{array}{c}
\frac{5}{4}+\frac{l-\rho}{2}, \frac{7}{4}+\frac{l-\rho}{2}, \frac{5}{4}+m+\frac{l-\rho}{2}, \frac{5}{4}-m+\frac{l-\rho}{2} \\
\frac{3}{4}+\frac{n}{2}, \frac{5}{4}+\frac{n}{2}, \frac{3}{4}-\frac{n}{2}, \frac{5}{4}-\frac{n}{2}: e^{i \pi} z^{2} \\
\frac{3}{2}, \frac{7}{4}+k+\frac{l-\rho}{2}, \frac{7}{4}+k-\frac{l+\rho}{2}
\end{array}\right]
$$

where

$$
R(\rho+n)>0, R\left(\frac{3}{2}-\rho+l\right)>0, R\left(\frac{3}{2} \pm 2 m+l-\rho\right)>0, R\left(\frac{5}{2}-\rho\right)>0
$$

and $|\operatorname{amp} z|<\pi$.

(14) in combination with (19) gives

$$
\begin{aligned}
& \int_{0}^{\infty} e^{-\mu} \mu^{\rho+l-1} I_{n}(\mu) W_{k_{1} m}(2 z \lambda) W_{-k_{1} m}(2 z \lambda) d \lambda= \\
& \frac{z^{-\rho-n-l} \Gamma\left(\frac{l+\rho+n+1}{2}\right) \Gamma\left(\frac{\rho+l+n+2}{2}\right) \Gamma\left(m+\frac{l+\rho+n+1}{2}\right)}{2 \sqrt{2} \Gamma\left(\frac{1}{2}\right) \Gamma\left(1+k+\frac{\rho+l+n}{2}\right)} \\
& \times \frac{\Gamma\left(-m+\frac{l+\rho+n+1}{2}\right) \Gamma\left(\frac{1}{4}+\frac{n}{2}\right) \Gamma\left(\frac{3}{4}+\frac{n}{2}\right)}{\Gamma\left(1+k+\frac{\rho+n+l}{2}\right) \Gamma(1+n) \Gamma\left(\frac{1}{2}+n\right)} \\
& \times_{6} F_{5}\left(\begin{array}{c}
\frac{l+\rho+n+1}{2}, \frac{l+\rho+n+2}{2}, m+\frac{l+\rho+n+1}{2},-m+\frac{l+\rho+n+1}{2}, \\
\frac{1}{4}+\frac{n}{2}, \frac{3}{4}+\frac{n}{2}, \frac{1}{4 z^{2}} \\
\frac{1}{2}, 1+k+\frac{\rho+l+n}{2}, 1+k+\frac{\rho+n-l}{2}, 1+n, \frac{1}{2}+n
\end{array}\right) \\
& +\frac{z^{-\rho-n-l} \Gamma\left(1+\frac{l+\rho+n}{2}\right) \Gamma\left(\frac{3}{2}+\frac{l+\rho+n}{2}\right) \Gamma\left(1+m+\frac{l+\rho+n}{2}\right)}{2 \sqrt{2} \Gamma\left(\frac{3}{2}\right) \Gamma\left(\frac{3}{2}+k+\frac{\rho+l+n}{2}\right)} \\
& \times \frac{\Gamma\left(1-m+\frac{l+\rho+n}{2}\right) \Gamma\left(\frac{3}{4}+\frac{n}{2}\right) \Gamma\left(\frac{5}{4}+\frac{n}{2}\right)}{\Gamma\left(\frac{3}{2}+k+\frac{\rho+n-l}{2}\right) \Gamma\left(\frac{3}{2}+n\right) \Gamma(1+n)} \\
& \times_{6} F_{5}\left(\begin{array}{c}
1+\frac{l+\rho+n}{2}, \frac{3}{2}+\frac{l+\rho+n}{2}, 1+m+\frac{l+\rho+n}{2}, 1-m+\frac{\rho+l+n}{2}, \\
\frac{3}{4}+\frac{n}{2}, \frac{5}{4}+\frac{n}{2} ; \frac{1}{4 z^{2}} \\
\frac{3}{2}, \frac{3}{2}+k+\frac{l+\rho+n}{2}, \frac{3}{2}+k+\frac{\rho+n-l}{2}, \frac{3}{2}+n, 1+n
\end{array}\right)
\end{aligned}
$$


where

$$
R(n+\rho+1+l)>0, R(n+\rho+1 \pm 2 m+l)>0, R(n+\rho+2)>0
$$

and $z$ is real and positive with $\left|4 z^{2}\right|>1$.

It may be noted that the formulae (50), (51), (53) can be generalized by applying (7)

\section{$\S 5$. Integrals involving the product of four Whittaker functions.}

(4) in combination with (2) gives

$$
\begin{aligned}
& \int_{0}^{\infty} t^{\rho-1} W_{k_{1} m}(t) W_{-k_{1} m}(t) W_{\mu, \nu}\left(\frac{2 i z}{t}\right) W_{\mu, \nu}\left(\frac{-2 i z}{t}\right) d t \\
= & \frac{2^{\rho-2 \mu-1} z^{2 \mu}}{\pi \Gamma\left(\frac{1}{2}-\mu+\nu\right) \Gamma\left(\frac{1}{2}-\mu-\nu\right)} \\
E & {\left[\begin{array}{c}
\frac{1}{2}-\mu+\nu, \frac{1}{2}-\mu-\nu, \frac{1}{2}-\mu, 1-\mu, \frac{1}{2}+\frac{1}{2} \rho-\mu+m, \\
\frac{1}{2}+\frac{1}{2} \rho-\mu-m, \frac{\rho+1}{2}-\mu, 1+\frac{\rho}{2}-\mu: \frac{z^{2}}{4} \\
1-2 \mu, 1+\frac{1}{2} \rho-\mu-k, 1+\frac{1}{2} \rho-\mu+k
\end{array}\right] }
\end{aligned}
$$

where

$$
R(\rho-2 \mu+2 m)>-1, R(\rho)>-1 \text { and } \mid \text { amp } z \mid<\pi .
$$

(19) in combination with (2) gives

$$
\begin{gathered}
\int_{0}^{\infty} t^{\rho-1} W_{k_{1} m}(t) W_{-k_{1} m}(t) W_{\mu, \nu}(2 z t) W_{-\mu, \nu}(2 z t) d t=\frac{2^{\rho+l-1}}{2 \pi^{2} z^{l}} \\
\times \sum_{i,-i} \frac{1}{i} E\left[\begin{array}{c}
\frac{l+1}{2}, 1+\frac{l}{2}, 1, \frac{l+1}{2}+\nu, \frac{l+1}{2}-\nu, \frac{l+\rho+1}{2}+m, \\
\frac{l+\rho+1}{2}+m, \frac{l+\rho+1}{2}, 1+\frac{l+\rho+1}{2}, 1+\frac{l+\rho}{2}: e^{i \pi} \frac{z^{2}}{4} \\
1+\mu+\frac{l}{2}, 1+\mu-\frac{l}{2}, 1+\frac{1}{2} l+\frac{1}{2} \rho-k, 1+\frac{1}{2} l+\frac{1}{2} \rho+\frac{1}{2} k
\end{array}\right] .
\end{gathered}
$$

where

$$
R(\rho \pm 2 m)>-1, R(\rho)>-1,|\operatorname{amp} z|<\pi
$$

It may be noted that the parameter $l$ does not appear on the left side of (56). Also (56) can be generalized by (7). 
$\S 6$. Integrals involving the product of an exponential function and 3 three Whittaker functions:

(1) in combination with (2) gives

$$
\begin{aligned}
& \int_{0}^{\infty} t^{\rho-1} e^{\frac{z^{2}}{2 t^{2}}} W_{k_{1} m}(t) W_{-k_{1} m}(t) W_{\mu, \nu}\left(\frac{z^{2}}{t^{2}}\right) d t \\
&= \frac{2^{\rho-2 \mu-1} z^{2 k}}{\Gamma\left(\frac{1}{2}\right) \Gamma\left(\frac{1}{2}-\mu-\nu\right) \Gamma\left(\frac{1}{2}-\mu-\nu\right)} \\
& \times E\left[\begin{array}{c}
\frac{1}{2}-\mu+\nu, \frac{1}{2}-\mu-\nu, \frac{\rho+1}{2}-\mu+m, \frac{\rho+1}{2}-\mu-m, \\
1+\frac{1}{2} \rho-\mu, \frac{1}{2}+\frac{1}{2} \rho-\mu: \frac{z^{2}}{4} \\
1+\frac{1}{2} \rho-\mu+k, 1+\frac{1}{2} \rho-\mu-k
\end{array}\right]
\end{aligned}
$$

where

$$
R(\rho \pm 2 m)>1, R(\rho)>-1,|\operatorname{amp} z|<\frac{\pi}{2}
$$

(15) in combination with (2) gives

$$
\begin{array}{r}
\int_{0}^{\infty} t^{\rho-1} \exp \left(-\frac{z^{2}}{2 t^{2}}\right) W_{k_{1} m}(t) W_{-k_{1} m}(t) W_{\mu, \nu}\left(\frac{z^{2}}{t^{2}}\right) d t=\frac{z^{-2 l} 2^{\rho+2 l-2}}{\sqrt{\pi}} \\
\frac{1}{2 \pi} \sum_{i,-i} \frac{1}{i} E\left[\begin{array}{c}
\frac{1}{2}+\nu+l, \frac{1}{2}-\nu+l, 1, \frac{\rho+1}{2}+l+m, \frac{\rho+1}{2}+l-m, \\
\frac{\rho+1}{2}+l, 1+\frac{\rho}{2}+l: e^{i \pi} \frac{z^{2}}{4} \\
1-\mu+l, 1+l+\frac{1}{2} \rho-k, 1+\frac{1}{2} \rho+l+k
\end{array}\right]
\end{array}
$$

where

$$
R(\rho \pm 2 m)>-1, R(\rho)>1,|\operatorname{amp} z|<\frac{\pi}{2}
$$

It may be noted that $l$ does not appear on the left side of (60). Also (60) can be generalized by (7).

When $l=\mu$, the formula becomes

$$
\begin{gathered}
\int_{0}^{\infty} \exp \left(\frac{-z^{2}}{2 t^{2}}\right) t^{\rho-1} W_{k_{1} m}(t) W_{-k_{1} m}(t) W_{\mu, \nu}\left(\frac{z^{2}}{t^{2}}\right) d t=\frac{z^{-2 \mu}}{\sqrt{\pi}} \\
\frac{2^{2 \rho+2 \mu-1}}{\sqrt{\pi}} \sum_{i,-i} \frac{1}{i} E\left[\begin{array}{c}
\frac{1}{2}+\nu+\mu, \frac{1}{2}-\nu+\mu, \frac{\rho+1}{2}+\mu+m, \frac{\rho+1}{2}+\nu-m, \\
\frac{\rho+1}{2}+\mu, 1+\frac{\rho}{2}+\mu: e^{i \pi} \frac{z^{2}}{4} \\
1+\mu+\frac{1}{2} \rho-k, 1+\mu+k+\frac{1}{2} \rho
\end{array}\right]
\end{gathered}
$$


wher

$$
R(\rho \pm 2 m)>1, R(\rho)>1,|\operatorname{amp} z|<\frac{\pi}{2} .
$$

Also (61) can generalized by (7).

\section{References}

[1] MacRobert T.M., "Functions of a Complex Variable" 4th edit. London (1954).

[2] Ragab, F.M., Journal of the London Mathematical Society 37 (1962).

[3] — - "Some formulae for the product of two Whittaker Functions", Konimble Akademie Van Wetenschappen, Amsterdam Vol. (58), pp. 430-434, (1958).

[4] Ragab F.M., "Integrals involving products of Bessel functions", Annali di Matematica V. LVI, pp. 301-312, (1961).

[5] MacRobert T.M. "Moltiplication formulae for the $E$ functions", Pacifie journal of Math. Vol. 9, pp. 759-761, (1959).

[6] Ragab F.M. Proc. Glay, Math. Assoc. II, (1954).

[7] MacRobert T.M., Philos Magan Ser. 7. XXXI (1941).

[8] Ragab F.M. Proc. Glag. Math. Assoc İ, (1951).

[9] - Proc. Glasgow. Math Assoc. II (1953).

[10] —, Proc. Glasgow, Math. Ass. II. (1954).

Mathematic Department, Faculty of Engineering, Cairo University. Egypt. A.R.E. 